\title{
Fibre supplementation for the prevention of type 2 diabetes and improvement of glucose metabolism: the randomised controlled Optimal Fibre Trial (OptiFiT)
}

\author{
Caroline Honsek $^{1}$ - Stefan Kabisch ${ }^{1,2}$ - Margrit Kemper ${ }^{1,2} \cdot$ Christiana Gerbracht $^{1}$ - Ayman M. Arafat ${ }^{1,3}$. \\ Andreas L. Birkenfeld ${ }^{2,4,5}$. Ulrike Dambeck ${ }^{1}$ - Martin A. Osterhoff ${ }^{1,3} \cdot$ Martin O. Weickert ${ }^{6,7,8} \cdot$ Andreas F. H. Pfeiffer $^{1,2,3}$
}

Received: 21 August 2017 / Accepted: 17 January 2018 / Published online: 28 February 2018

(C) The Author(s) 2018. This article is an open access publication

\begin{abstract}
Aims/hypothesis Insoluble cereal fibres have been shown in large prospective cohort studies to be highly effective in preventing type 2 diabetes, but there is a lack of interventional data. Our 2 year randomised double-blind prospective intervention study compared the effect of an insoluble oat fibre extract with that of placebo on glucose metabolism and incidence of diabetes.

Methods A total of 180 participants with impaired glucose tolerance underwent a modified version of the 1 year lifestyle training programme PREvention of DIAbetes Self-management (PREDIAS) and were randomised to receive a fibre supplement ( $n=89 ; 7.5 \mathrm{~g}$ of insoluble fibre per serving) or placebo $(n=91 ; 0.8 \mathrm{~g}$ of insoluble fibre per serving) twice daily for 2 years. Eligible participants were men and women, were at least 18 years old and did not report corticosteroid or other intensive anti-inflammatory treatment, fibre intolerance or any of the following disorders: overt diabetes, chronic or malignant disease, or severe cardiopulmonary, endocrine, psychiatric, gastrointestinal, autoimmune or eating disorder. Participants were recruited at two clinical wards in Berlin and Nuthetal. The allocation was blinded to participants and study caregivers (physicians, dietitians, study nurses). Randomisation was conducted by non-clinical staff, providing neutrally numbered supplement tins. Both supplements were similar in their visual, olfactory and gustatory appearance. Intention-to-treat analysis was applied to all individuals.

Results After 1 year, $2 \mathrm{~h}$ OGTT levels decreased significantly in both groups but without a significant difference between the groups (fibre $-0.78 \pm 1.88 \mathrm{mmol} / 1[p \leq 0.001]$ vs placebo $-0.46 \pm 1.80 \mathrm{mmol} / 1[p=0.020]$; total difference $0.32 \pm 0.29 \mathrm{mmol} / \mathrm{l}$;
\end{abstract}

Caroline Honsek and Stefan Kabisch contributed equally to this publication.

Electronic supplementary material The online version of this article (https://doi.org/10.1007/s00125-018-4582-6) contains peer-reviewed but unedited supplementary material, which is available to authorised users.

Stefan Kabisch

Stefan.kabisch@dife.de

$\triangle$ Martin O. Weickert

Martin.Weickert@uhcw.nhs.uk

1 Department of Clinical Nutrition, German Institute of Human Nutrition Potsdam-Rehbrücke, Arthur-Scheunert-Allee 114-116, 14558 Nuthetal, Germany

2 German Center for Diabetes Research (DZD), München-Neuherberg, Germany

3 Department of Endocrinology, Diabetes and Nutrition, Campus Benjamin Franklin, Charité University Medicine, Berlin, Germany

4 Section of Metabolic Vascular Medicine, Medical Clinic III and Paul Langerhans Institute Dresden of the Helmholtz Center Munich at University Hospital and Faculty of Medicine, Dresden, Germany
5 Studienzentrum für Metabolisch-Vaskuläre Medizin, Gesellschaft für Wissens- und Technologietransfer TU Dresden (GWT-TUD), Dresden, Germany

6 Warwickshire Institute for the Study of Diabetes, Endocrinology and Metabolism, The ARDEN NET Centre, European Neuroendocrine Tumor Society Center of Excellence (ENETS CoE), University Hospitals Coventry and Warwickshire NHS Trust, Clifford Bridge Road, Coventry CV2 2DX, UK

7 Centre of Applied Biological and Exercise Sciences (ABES), Faculty of Health and Life Sciences, Coventry University, Coventry, UK

Translational and Experimental Medicine, Division of Biomedical Sciences, Warwick Medical School, University of Warwick, Coventry, UK 


\section{Research in context}

\section{What is already known about this subject?}

- $\quad$ Large prospective cohort studies have shown insoluble cereal fibre to be highly effective in preventing type 2 diabetes, but there is a lack of interventional data

\section{What is the key question?}

- Is 2 year supplementation with insoluble oat fibre extract, compared with placebo, superior with regard to glucose metabolism and incidence of diabetes?

\section{What are the new findings?}

- Two hour OGTT levels and insulin resistance decreased significantly and similarly in both groups, with a decreasing effect size after 2 years

- $\quad \mathrm{HbA}_{1 \mathrm{c}}$ levels decreased significantly more in the fibre group

- Separate analysis of the sexes revealed a significantly greater reduction of $2 \mathrm{~h}$ glucose levels for women in the fibre group

\section{How might this impact on clinical practice in the foreseeable future?}

- Despite failing to provide strong evidence for a benefit of insoluble cereal fibre on glycaemic metabolism, further studies may support minor effects of fibre supplementation in the reduction of glucose levels, insulin resistance and incidence of type 2 diabetes

not significant). The 2 year incidence of diabetes was 9/89 (fibre group) compared with 16/91 (placebo group; difference not significant). As secondary outcomes, the change in $\mathrm{HbA}_{1 \mathrm{c}}$ level was significantly different between the two groups $(-0.2 \pm 4.6 \mathrm{mmol} / \mathrm{mol}[-0.0 \pm 0.0 \%$; not significant $]$ vs $+1.2 \pm 5.2 \mathrm{mmol} / \mathrm{mol}[+0.1 \pm 0.0 \%$; not significant $]$; total difference $1.4 \pm 0.7 \mathrm{mmol} / \mathrm{mol}[0.1+0.0 \%]) ; p=0.018)$; insulin sensitivity and hepatic insulin clearance increased in both groups. After 2 years, improved insulin sensitivity was still present in both groups, although the effect size had diminished. Separate analysis of the sexes revealed a significantly greater reduction in $2 \mathrm{~h}$ glucose levels for women in the fibre group $(-0.88 \pm 1.59 \mathrm{mmol} / 1[p \leq 0.001]$ vs $-0.22 \pm 1.52 \mathrm{mmol} / 1[p=0.311]$; total difference $0.67 \pm 0.31 \mathrm{mmol} / 1 ; p=0.015)$. Levels of fasting glucose, adipokines and inflammatory markers remained unchanged in the two groups. Significantly increased fibre intake was restricted to the fibre group, despite dietary counselling for both groups. No severe side effects occurred.

Conclusions/interpretation We cannot currently provide strong evidence for a beneficial effect of insoluble cereal fibre on glycaemic metabolism, although further studies may support minor effects of fibre supplementation in reducing glucose levels, insulin resistance and the incidence of type 2 diabetes.

Trial registration: clinicaltrials.gov NCT01681173

Funding: German Diabetes Foundation (grant no. 232/11/08)

Keywords Cellulose $\cdot$ Diabetes mellitus type $2 \cdot$ Hemicellulose $\cdot$ Hepatic insulin clearance $\cdot$ Impaired glucose tolerance $\cdot$ Insoluble dietary fibre $\cdot$ Insulin sensitivity $\cdot$ Prediabetes

\author{
Abbreviations \\ BIA Bioelectrical impedance analysis \\ CRP C-reactive protein \\ HIC Hepatic insulin clearance \\ IGT Impaired glucose tolerance \\ OptiFiT Optimal Fibre Trial for Diabetes Prevention \\ PREDIAS PREvention of DIAbetes Self-management \\ $\mathrm{Sg} \quad$ Glucose effectiveness index
}

\section{Introduction}

The effectiveness of preventing type 2 diabetes mellitus by moderate alterations in lifestyle has been proven in two previous trials [1, 2]. Additionally, large epidemiological studies have identified dietary fibre as one of the most effective nutrient components for diabetes prevention [3]. Fibre intake is currently far below the recommended 
levels in most countries that consume Western-style foods [4]. According to epidemiological data, the preventive effect of fibres is most evident with cereal but not at all with fruit, legumes and vegetable fibres [5, 6]. Cereals contain predominantly insoluble fibres from the coat region and a smaller fraction of soluble fibres from the endosperm and aleurone layers of the grain. The insoluble fraction is composed of cellulose, hemicelluloses and lignin, and is only modestly fermented, such that the greater part is excreted with the faeces [7]. This modest fermentation leads to little gas production in the intestine, making insoluble fibres more acceptable to people who are sensitive to bowel symptoms.

Mechanisms linking insoluble fibres to glucose homeostasis in humans point to a rapid, overnight improvement in meal-related insulin sensitivity without significant changes in insulin secretion when using either insoluble fermentable or non-fermentable fibres or whole grain $[8,9]$. Using cereal fibre extracts identical to those used in the current study, we have shown an improvement in euglycaemichyperinsulinaemic-clamp-measured whole-body insulin sensitivity after 3 days and 6 weeks in humans [10,11], as well as beneficial metabolic effects in a long-term controlled study in C57BL/6J mice [7].

Long-term prospective randomised data on fibre supplementation in humans are sparse, and available data do not show striking changes in glycaemic variables. A 1 year study involving 14 participants failed to demonstrate beneficial effects, possibly due to relatively small differences in fibre intake [12]. So far, no evidence for an interventional effect of soluble fibre on glucose levels has been provided [13-15].

Recent diabetes prevention trials have shown that lifestyle intervention reduces the risk of incident diabetes by 40-60\% compared with placebo [1, 2, 16, 17]. These studies targeted primarily weight loss and increased physical activity. Other studies have investigated the effect of a reduced intake of saturated and total fat and increased fibre intake as part of daily nutrition. However, relevantly increased fibre intake was usually not achieved [18]. Therefore, supplementation appears to be a more efficient way to increase fibre consumption. Here, we hypothesised that a continuous increase in fibre supplementation of everyday food intake would lead to a significant reduction in the incidence of type 2 diabetes in a high-risk cohort. In order to select and design foods containing effective fibre types, and to estimate the effect size of increased fibre intake, proof-of-principle studies are needed. We therefore decided to perform a long-term prospective randomised placebo-controlled trial investigating whether insoluble cereal fibres would improve glucose metabolism over 12 and 24 months in individuals with impaired glucose tolerance (IGT).

\section{Methods}

Study population Men and women with IGT were recruited to the Optimal Fibre Trial for Diabetes Prevention (OptiFiT) between March 2010 and October 2012, and enrolled at two study sites (German Institute of Human Nutrition, PotsdamRehbrücke, and Department of Endocrinology and Metabolic Diseases, Charité University Medicine, Berlin). The participants were recruited via an existing database, public advertisement, word of mouth, radio advertisements and flyers. A total of 652 participants were screened using an OGTT, and 180 participants with IGT were enrolled into the study. IGT was defined as a $2 \mathrm{~h}$ plasma glucose concentration of $7.8-$ $11.1 \mathrm{mmol} / \mathrm{l}$.

According to the inclusion criteria, eligible study volunteers were aged $\geq 18$ years and were willing to participate in one of the two intervention groups over a period of 2 years. Exclusion criteria were a history of type 1 or type 2 diabetes, pregnancy, breastfeeding or plans to become pregnant in the next 2 years, chronic or malignant diseases, other wasting illnesses (e.g. severe cardiopulmonary or autoimmune disorders), or a history or presence of a significant psychiatric disorder, eating disorders, medication with systemic corticosteroids, food allergies or food intolerances that precludes a supplementation with dietary fibre, and any other condition that would interfere with participation in the study.

The study protocol was approved by the ethics committee of the University of Potsdam as well as the ethics committee of the Charité, and all individuals gave written informed consent. The trial was registered at clinicaltrials.gov (NCT 01681173).

Study design OptiFiT is a randomised, placebo-controlled, double-blind, parallel-group 24 month intervention study. Volunteers were randomly assigned using a computer algorithm to either placebo or fibre supplement by office staff who were not involved in other study procedures. Study enrolment was carried out by four study physicians, and dietary intervention was conducted by the nutritionist. Every 6 months, anthropometric data and serum markers were assessed, and all participants repeated the OGTT after 12 and 24 months.

In addition to having the dietary supplement, all participants started the 24 month study with a modified 1 year lifestyle programme, based on PREvention of DIAbetes Self-management (PREDIAS), a structured 'Treatment and Education Program for Prevention of type 2 diabetes' $[1,19]$. Within this course, the volunteers had groupbased consultations at regular intervals. During the second year of the study, the participants continued receiving their study supplement and were asked to continue regular intake twice daily. 
Dietary intervention The 12 month lifestyle PREDIAS consisted of 12 individual $2 \mathrm{~h}$ lessons divided into a core intervention (eight lessons in 8 weeks) and booster sessions (four lessons throughout the following 10 months) [20]. With this cost-effective self-management approach, participants should learn how to achieve a healthy balanced diet and adequate physical activity. For our modified lifestyle intervention, participants completed food records for 4 consecutive days, including 1 weekend day, before the study and after 6, 12 and 24 months. They were advised to record everything they ate and drank, and the completeness of the food records was checked with the study nutritionist. Nutrient intake was determined using nutrition software PRODI 5.8 (Wissenschaftliche Verlagsgesellschaft, Stuttgart) based on Bundeslebensmittelschlüssel 3.0 [21].

All participants were given detailed advice about how to achieve the goals of the lifestyle intervention: an increase in fibre intake to at least $15 \mathrm{~g}$ per $4200 \mathrm{~kJ}$; a reduction in weight of $5 \%$ or more (if baseline BMI $>25 \mathrm{~kg} / \mathrm{m}^{2}$ ); a reduction in total intake of fat to less than $30 \%$ of energy consumed and of saturated fat to less than $10 \%$ of energy consumed; and moderate exercise for at least $30 \mathrm{~min}$ per day. Frequent ingestion of whole-grain products, legumes, vegetables, fruits, in particular berries, low-fat milk and meat products, soft margarines, and vegetable oils rich in unsaturated fatty acids was recommended. At regular intervals, all participants received a pedometer to assess their physical activity.

Dietary supplements The supplements were provided as drinking powders every 6 months, when the individuals attended the metabolic unit, and participants were instructed to take the supplements twice daily. To enrich the high-fibre supplement with insoluble cereal fibre, a purified fibre extract derived from oat hulls was used, which contained $70 \mathrm{wt} \%$ cellulose, $25 \mathrm{wt} \%$ hemicellulose and 3-5 wt\% lignin (Vitacel OF 560-30; Rettenmaier \& Söhne, Holzmuehle, Germany). Further details on the supplement composition can be found elsewhere [8]. In addition to this main component, the supplement contained guar gum (1.92 wt\%), isomaltulose, sucralose, flavouring and dyes in small amounts. This supplement added $15 \mathrm{~g}$ of mainly insoluble fibre per day $(7.5 \mathrm{~g}$ per serving) to the normal nutrition. The appearance of the placebo supplement was comparable, but instead of the high-fibre extract, a waxy maize starch with negligible content of insoluble fibre and guar gum $(0.8 \mathrm{~g}$ and $0.4 \mathrm{~g}$ per serving, respectively) and isomaltulose was used.

The fibre and placebo supplements contained 251 and $276 \mathrm{~kJ}$ per serving, respectively, had a comparable fruity taste and did not differ in appearance or texture. Blinding applied to both participants and study personnel. The volunteers were provided with instructions on how to use the drinking powder: the recommended intake was twice daily dissolved in $300 \mathrm{ml}$ water, preferably before breakfast and dinner. The participants received accurately weighed quantities of the supplement in individual tins and were asked to return the amount they did not consume during their 6-monthly visits to the study unit.

Measurements and laboratory variables The primary outcome was the change in $2 \mathrm{~h}$ post-challenge plasma glucose in the annual $75 \mathrm{~g}$ OGTT after a $12 \mathrm{~h}$ fast. IGT and diabetes were defined according to the recommendations of the ADA, IDF and WHO: participants with IGT had plasma glucose levels between 7.8 and $11.1 \mathrm{mmol} / \mathrm{l}$, and a glucose value above that range was an indicator of diabetes. If diabetes was diagnosed during the study, the participants were referred to their general physician for treatment, and follow-up in OptiFiT was discontinued.

Blood samples for the OGTT were taken before and at 60, 120 and 180 min to measure glucose, insulin and Cpeptide. Capillary blood glucose concentrations were measured immediately using the glucose oxidase method (Super-GL glucose analyser; Dr Müller Gerätebau, Freital, Germany); venous serum blood samples were analysed batch-wise after storage (Horiba ABX Pentra 400, Montpellier, France). Serum insulin and C-peptide were measured using an ELISA technique (Mercodia, Uppsala, Sweden).

In addition to the annual OGTT, routine laboratory markers were measured every 6 months by standard methods in the research laboratories of the German Institute of Human Nutrition. After sampling in EDTA or serum tubes, blood was immediately chilled on ice and centrifuged, and aliquots were immediately frozen at $-80^{\circ} \mathrm{C}$ until assayed. Blood samples were analysed for total cholesterol, LDL- and HDL-cholesterol, triacylglycerols, NEFA, C-reactive protein, liver enzymes and $\mathrm{HbA}_{1 \mathrm{c}}$ levels (ABX Pentra 400; Horiba ABX).

The baseline and 6-monthly clinical examinations included measurements of weight to the nearest $0.1 \mathrm{~kg}$ (in light indoor clothes without shoes), height to the nearest $0.5 \mathrm{~cm}$ and waist and hip circumference. Fat mass was assessed by bioelectrical impedance analysis (BIA; Body Impedance Analyser Quantum/S, Akern Bioresearch, Florence, Italy).

Calculations The AUC in response to the $75 \mathrm{~g}$ OGTT was calculated for plasma glucose, insulin and C-peptide to combine multiple readings into a single index by the trapezoidal method. Insulin resistance was estimated by calculating the following fasting indices: HOMA-IR [22], QUICKI [23] and insulin sensitivity index of blood NEFA [24]. Additionally, dynamic insulin sensitivity indices-glucose effectiveness $\mathrm{Sg}$ [25] and the Cederholm 
Table 1 Characteristics of participants at study entry

\begin{tabular}{|c|c|c|}
\hline Variable & Placebo $(n=91)$ & Fibre $(n=89)$ \\
\hline \multicolumn{3}{|l|}{ Sex, $n(\%)$} \\
\hline Male & $37(41 \%)$ & $23(26 \%)^{\dagger}$ \\
\hline Female & $54(59 \%)$ & $66(74 \%)^{\dagger}$ \\
\hline Age (years) & $60 \pm 10$ & $59 \pm 10$ \\
\hline \multicolumn{3}{|l|}{ Smoking status $(\%)$} \\
\hline Current & 4.4 & 6.7 \\
\hline Former & 60.4 & 67.4 \\
\hline Never & 35.2 & 25.8 \\
\hline \multicolumn{3}{|l|}{ Family history (\%) } \\
\hline Hypertension & 67.4 & 61.4 \\
\hline Type 2 diabetes & 69.2 & 69.4 \\
\hline Type 1 diabetes & 4.4 & 1.1 \\
\hline \multicolumn{3}{|l|}{ Blood pressure (mmHg) } \\
\hline Systolic & $138 \pm 17$ & $140 \pm 21$ \\
\hline Diastolic & $87 \pm 11$ & $90 \pm 13$ \\
\hline Weight (kg) & $92.3 \pm 20.7$ & $88.1 \pm 15.2$ \\
\hline BMI $\left(\mathrm{kg} / \mathrm{m}^{2}\right)$ & $33.0 \pm 6.4$ & $31.8 \pm 5.3$ \\
\hline Height (cm) & $167 \pm 9$ & $167 \pm 8$ \\
\hline Hip circumference $(\mathrm{cm})$ & $114.0 \pm 13.3$ & $111.9 \pm 12.2$ \\
\hline Waist circumference $(\mathrm{cm})$ & $105.5 \pm 14.4$ & $103.5 \pm 12.3$ \\
\hline WHR & $0.93 \pm 0.1$ & $0.93 \pm 0.1$ \\
\hline Body fat mass ${ }^{\mathrm{a}}(\%)$ & $38.3 \pm 9.1$ & $37.0 \pm 8.6$ \\
\hline Fasting glucose (mmol/l) & $6.0 \pm 0.6$ & $5.9 \pm 0.7$ \\
\hline $2 \mathrm{~h}$ glucose $(\mathrm{mmol} / \mathrm{l})$ & $9.2 \pm 1.8$ & $9.0 \pm 1.7$ \\
\hline $\mathrm{HbA}_{1 \mathrm{c}}(\mathrm{mmol} / \mathrm{mol})$ & $38.0 \pm 4.2$ & $38.4 \pm 4.2$ \\
\hline $\mathrm{HbA}_{1 \mathrm{c}}(\%)$ & $5.6 \pm 0.4$ & $5.7 \pm 0.4$ \\
\hline Insulin (pmol/l) & $70.1 \pm 38.2$ & $62.5 \pm 31.9$ \\
\hline C-peptide (nmol/1) & $0.57 \pm 0.27$ & $0.57 \pm 0.33$ \\
\hline $\mathrm{HIC}_{\mathrm{C}-\text { peptide }}(\mathrm{pmol} / \mathrm{nmol})$ & $3.2 \pm 1.3$ & $3.1 \pm 1.2$ \\
\hline Total cholesterol (mmol/l) & $5.6 \pm 0.9$ & $5.6 \pm 1.1$ \\
\hline HDL-cholesterol (mmol/l) & $1.3 \pm 0.3$ & $1.2 \pm 0.2$ \\
\hline LDL-cholesterol (mmol/l) & $3.5 \pm 0.8$ & $3.7 \pm 0.9$ \\
\hline CRP (nmol/l) & $35.1 \pm 37.1$ & $45.0 \pm 46.7$ \\
\hline Aspartate aminotransferase (U/l) & $28 \pm 16$ & $26 \pm 7$ \\
\hline Alanine aminotransferase (U/l) & $33 \pm 25$ & $27 \pm 11$ \\
\hline$\gamma$-Glutamyl transferase(U/l) & $31 \pm 27$ & $32 \pm 31$ \\
\hline Creatinine $(\mu \mathrm{mol} / \mathrm{l})$ & $75.0 \pm 16.1$ & $78.5 \pm 9.1$ \\
\hline Uric acid $(\mu \mathrm{mol} / \mathrm{l})$ & $341 \pm 84$ & $334 \pm 77$ \\
\hline
\end{tabular}

Data are mean $\pm \mathrm{SD}$

${ }^{a}$ Body fat mass measured by BIA

Significant difference between the groups: ${ }^{\dagger} p \leq 0.05$

index [26] - integrating plasma glucose and insulin concentrations during the OGTT, were used.

There is increasing evidence that hyperinsulinaemia in the insulin-resistant state reflects two pathophysiological processes: an increase in insulin secretion and a decrease in hepatic insulin clearance (HIC) [27]. Based on the

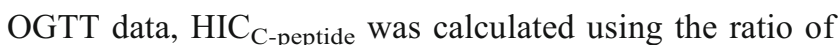
incremental areas under the insulin and $\mathrm{C}$-peptide curve:

$\mathrm{HIC}_{\mathrm{C} \text {-peptide }}=\mathrm{AUC}_{\mathrm{C} \text {-peptide }}[\mathrm{nmol} / \mathrm{l}] / \mathrm{AUC}_{\text {Insulin }}[\mathrm{pmol} / \mathrm{l}]$

Statistical analyses The power calculation was based on an estimated effect size of $0.39 \mathrm{mmol} / \mathrm{l}$ difference in $2 \mathrm{~h}$ OGTT blood glucose levels (primary outcome) between the two groups, an SD of $1.1 \mathrm{mmol} / 1$ and $80 \%$ power to detect the effect. Such effects have recently been reported in other lifestyle intervention studies $[2,19]$. The estimated number of participants to show these effects was 252 , excluding a $25 \%$ allowance for dropouts $(n=63)$.

Changes in fasting glucose, $\mathrm{HbA}_{1 \mathrm{c}}$, body weight, measures of insulin sensitivity and insulin secretion, inflammation and adipokines were defined as secondary outcomes.

To ensure uniform representation, calculations within groups were performed assuming a non-normal distribution, and thus differences in the variables studied before and after the intervention within the groups were calculated by Wilcoxon test. Using parametric tests whenever appropriate did not lead to different overall results. For the comparison of changes between groups, the unpaired Student's $t$ test with an adjustment for change in body weight was used as far as data normality was given. The characteristics of the participants are presented as means $\pm \mathrm{SD}$. The results were considered significantly different if $p \leq 0.05$. Every analysis was conducted both as an intention-to-treat (last observation carried forward) and as a per-protocol (completers only) analysis. If not explicitly stated, intention-to-treat and per-protocol analysis did not lead to different results, and intention-to-treat data are presented.

All statistical analyses were performed using SPSS for Windows version 22.0 (SPSS, Chicago, IL, USA). The graphs were created using GraphPad Prism version 6.0 (GraphPad Software, San Diego, CA, USA).

\section{Results}

The study sample comprised 180 men and women with a mean age of $60 \pm 10$ years. The baseline characteristics of the two groups, including all measured risk factors for diabetes, were similar, except for sex distribution (Table 1), which was not a matched variable for inclusion or randomisation. A total of 136 participants completed the first study year and were included in the primary analysis; 14 individuals were diagnosed with type 2 diabetes before the 1 year visit, and 30 volunteers ( 17 from the fibre group) dropped out for various reasons (illness, non-acceptance of the supplement, lack of time). 

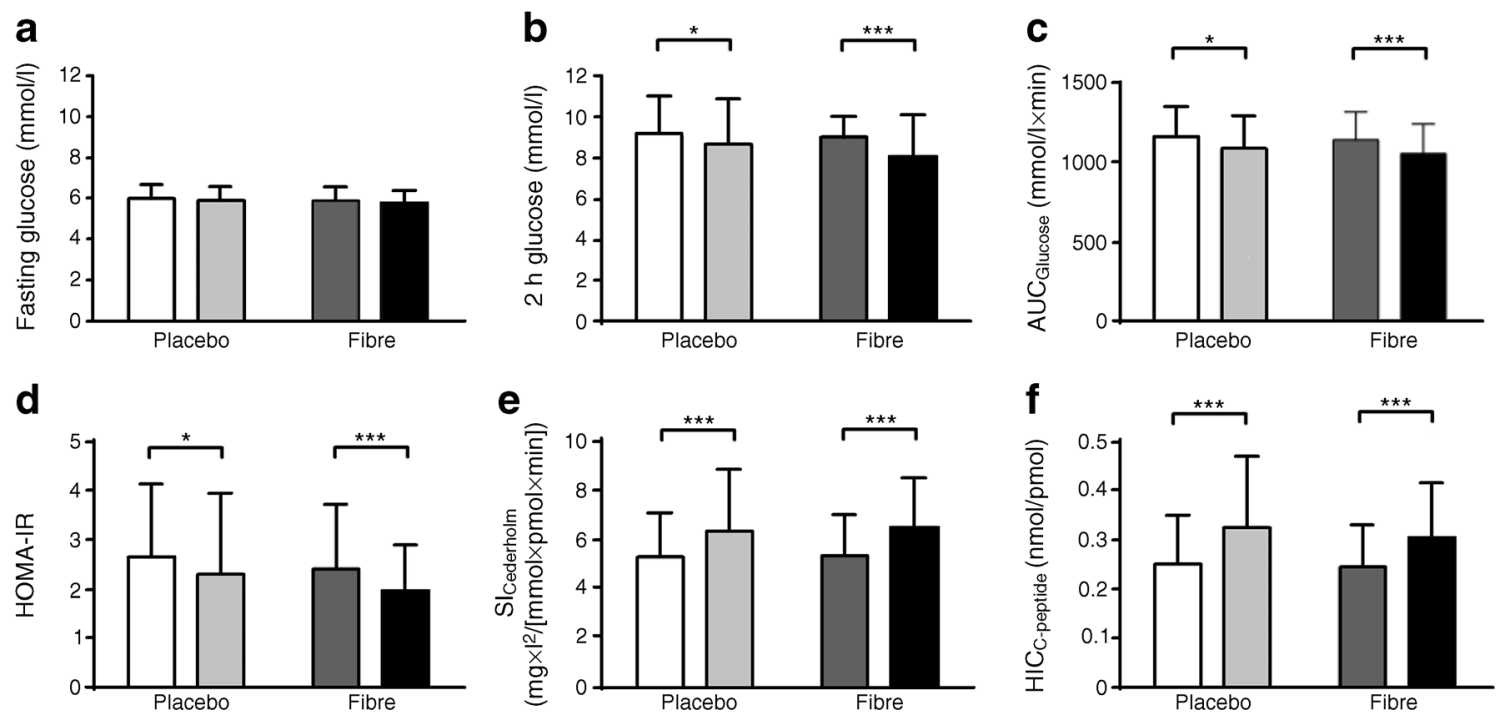

Fig. 1 Glycaemic control after 1 year of intervention (placebo group, $n=66$; fibre group, $n=64$ ). (a) Fasting glucose; (b) $2 \mathrm{~h}$ glucose; (c) $\mathrm{AUC}_{\mathrm{Glucose}}$; (d) fasting insulin sensitivity (HOMA-IR); (e) dynamic insulin sensitivity $\left(\mathrm{SI}_{\text {Cederholm}}\right)$; (f) $\mathrm{HIC}_{\mathrm{C} \text {-peptide }}$. White bars, placebo at

During the 12 months, mean body weight decreased by 2.0 $\pm 5.2 \mathrm{~kg}$ in the fibre group and by $2.6 \pm 5.2 \mathrm{~kg}$ in the placebo group (total difference $0.6 \pm 0.7 \mathrm{~kg}$; not significant). Variables such as BMI and waist and hip circumference significantly decreased in both groups, fat mass (BIA) decreased in the placebo group only, whereas blood pressure and WHR did not change in either group (see electronic supplementary material [ESM] Table 1).

The change in $\mathrm{HbA}_{1 \mathrm{c}}$ levels was significantly different between the two groups $(-0.2 \pm 4.6 \mathrm{mmol} / \mathrm{mol}[-0.0 \pm 0.0 \%$; not significant $] \mathrm{vs}+1.2 \pm 5.2 \mathrm{mmol} / \mathrm{mol}[+0.1 \pm 0.0 \%$; not baseline; light grey bars, placebo after 1 year of intervention; dark grey bars, fibre at baseline; black bars, fibre after 1 year of intervention. Data are mean \pm SD. Significant change within the group: $* p \leq 0.05$, *** $p \leq$ 0.001

significant]; total difference $1.4 \pm 0.7 \mathrm{mmol} / \mathrm{mol}[0.1+$ $0.0 \%] ; p=0.018$, adjusted for weight change).

In the fibre and placebo group, respectively, we found significantly decreased $2 \mathrm{~h}$ plasma glucose levels $(-0.78 \pm$ $1.88 \mathrm{mmol} / 1[p \leq 0.001]$ vs $-0.46 \pm 1.80 \mathrm{mmol} / 1[p=0.020]$; total difference $0.32 \pm 0.29 \mathrm{mmol} / \mathrm{l}$; not significant) and AUC for glucose $(-71.4 \pm 184.0 \mathrm{mmol} / 1 \times \min [p \leq 0.001] \mathrm{vs}-59.4$ $\pm 156.7 \mathrm{mmol} / 1 \times \min [p \leq 0.001]$, total difference $12.0+$ $27.0 \mathrm{mmol} / 1 \times \mathrm{min}$; not significant) without a significant difference between the groups. Fasting blood glucose did not change (Fig. 1a-c).
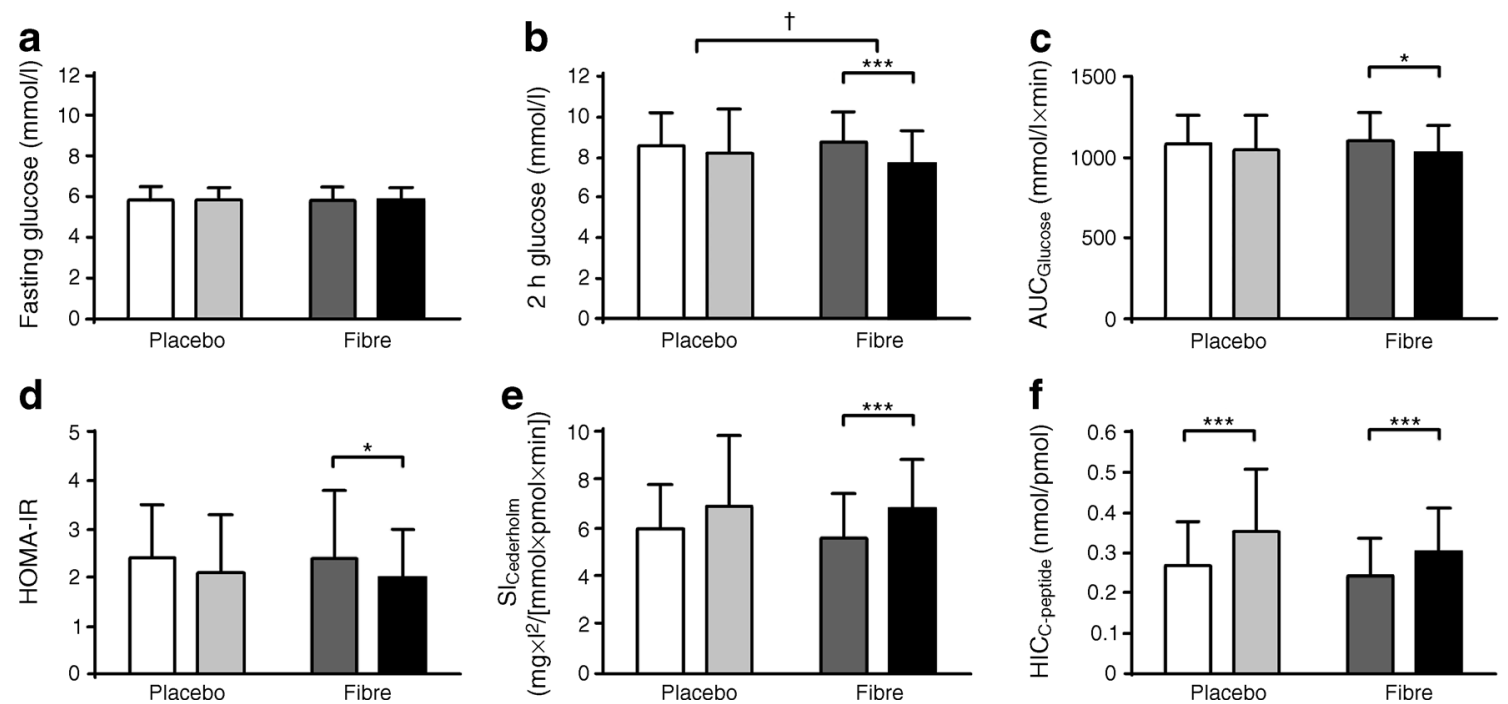

Fig. 2 Glycaemic control in women after 1 year of intervention (placebo group, $n=34$; fibre group, $n=47$ ). (a) Fasting glucose; (b) $2 \mathrm{~h}$ glucose; (c) $\mathrm{AUC}_{\mathrm{Glucose}}$; (d) fasting insulin sensitivity (HOMA-IR); (e)

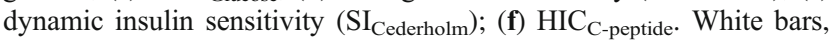

placebo at baseline; light grey bars, placebo after 1 year of intervention; dark grey bars, fibre at baseline; black bars, fibre after 1 year of intervention. Data are mean $\pm \mathrm{SD}$. Significant change within the group: ${ }^{*} p \leq 0.05$, $* * * p \leq 0.001$; significant change between groups: ${ }^{\dagger} p \leq 0.05$ 
Fig. 3 Mean dietary fibre intake without (a) and with (b) supplementation. White bars, placebo at baseline; light grey bars, placebo after 1 year of intervention; dark grey bars, fibre at baseline; black bars, fibre after 1 year of intervention. Data are mean \pm SD. Significant change within the group: $* * * p \leq 0.001$; significant change between groups: ${ }^{\dagger \dagger} p \leq 0.001$ a

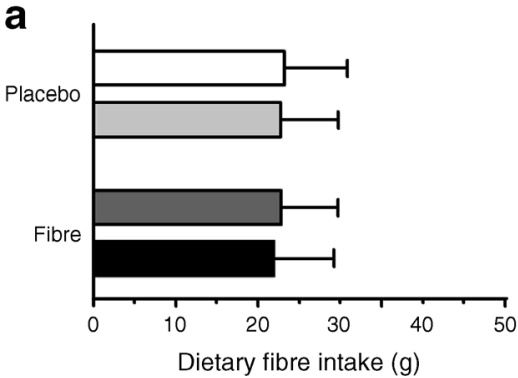

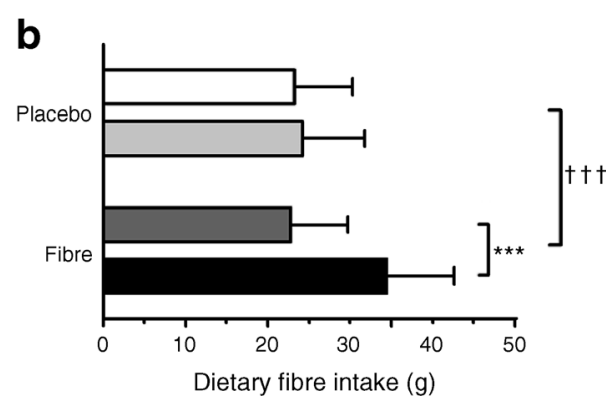

Fasting insulin levels significantly decreased only in the fibre group $(-9.1 \pm 22.2 \mathrm{pmol} / 1[p=0.001] \mathrm{vs}-6.3 \pm$ $29.9 \mathrm{pmol} / 1[p=0.064]$; total difference $2.8 \pm 4.2 \mathrm{pmol} / 1$ $[p=0.515])$. The insulin response after the glucose load was similar in the two groups. Additionally, $2 \mathrm{~h} \mathrm{C}$-peptide values fell significantly in both groups $(-0.2 \pm 0.8 \mathrm{nmol} / 1[p=0.018]$ vs $-0.3 \pm 1.1 \mathrm{nmol} / 1[p=0.045])$, although the 3 -h C-peptide value was only significantly reduced in the fibre group $(-0.2$ $\pm 0.6 \mathrm{nmol} / 1[p=0.013]$ vs $-0.1 \pm 0.7 \mathrm{nmol} / 1[p=0.504])$.

Fasting indices such as HOMA-IR and dynamic indices, combining insulin and glucose values during the OGTT, such as glucose effectiveness ( $\mathrm{Sg}$; data not shown) and Cederholm index, improved equally in the two groups (Fig. 1d, e). HIC improved similarly in both groups (Fig. 1f).

Further secondary outcomes revealed significant changes in C-reactive protein (CRP) (within both groups), IL-18 and chemerin (placebo group only), and leucocyte count and leptin (fibre group only), but the only significant difference between the groups was for leucocyte count (see electronic supplementary material [ESM] Table 1). During the first year of the intervention, the change in $\mathrm{AUC}_{\text {Glucose }}(r=0.199, p=0.114), \operatorname{HOMA}-\mathrm{IR}(r=$ $0.192, p=0.125)$ and Cederholm index $(r=-0.189, p=0.135)$ did not correlate with the weight change in the fibre group, but showed significant correlations in the placebo group: $\mathrm{AUC}_{\mathrm{Glucose}}$ $(r=0.250, p=0.043)$, HOMA-IR $(r=0.284, p=0.021)$ and Cederholm index $(r=-0.369, p=0.002)$.

Analysis for interaction with sex did not reveal significant results, but sex-specific analyses showed that, in women, only the fibre group achieved significant changes in $2 \mathrm{~h}$ plasma glucose $(-0.88 \pm 1.59 \mathrm{mmol} / 1[p \leq 0.001] \mathrm{vs}-0.22 \pm$ $1.52 \mathrm{mmol} / \mathrm{l}[p=0.311]), \mathrm{AUC}_{\text {Glucose }}(-65.4 \pm 175.1 \mathrm{mmol} /$ $1 \times \min [p=0.007] \mathrm{vs}-28.8 \pm 158.2 \mathrm{mmol} / 1 \times \min [p=$ $0.242]$ ), insulin levels (fasting, $2 \mathrm{~h}, 3 \mathrm{~h}$ and AUC; data not shown) and HOMA-IR $(-0.30 \pm 1.04[p=0.034]$ vs $-0.17 \pm$ $1.06[p=0.261])$. Of these, only the improvement in $2 \mathrm{~h}$ plasma glucose levels was significantly different between the fibre and placebo groups in women (total difference $0.67 \pm$ $0.31 \mathrm{mmol} / 1 ; p=0.015$, adjusted for weight change; Fig. 2). The relatively small and less balanced subgroup of men was not analysed separately.

One of the main goals of the lifestyle intervention course was a healthy high-fibre diet. The individual change in diet was assessed using food records, and the evaluation showed that, without the fibre supplement, the participants in both groups were not able to increase their fibre intake $(+0.9 \pm$ $8.0 \mathrm{~g}$ vs $+0.5 \pm 7.0 \mathrm{~g}$ ) despite advice to do so (Fig. 3). However, the participants in the fibre-supplemented group raised their average daily fibre intake from about
Table 2 Changes in dietary habits during the first year of the intervention

\begin{tabular}{|c|c|c|c|c|}
\hline \multirow[t]{2}{*}{ Variable } & \multicolumn{2}{|c|}{ Placebo $(n=59)$} & \multicolumn{2}{|l|}{ Fibre $(n=58)$} \\
\hline & Baseline & 12 months & Baseline & 12 months \\
\hline Total energy intake (kJ/day) & $8862 \pm 2536$ & $8205 \pm 2339^{*}$ & $8795 \pm 2059$ & $7598 \pm 1619 * * *$ \\
\hline Fat intake (g/day) & $82 \pm 28$ & $71 \pm 29^{*}$ & $80 \pm 26$ & $66 \pm 22 *$ \\
\hline Protein intake (g/day) & $84 \pm 23$ & $81 \pm 22$ & $81 \pm 24$ & $71 \pm 20^{*}$ \\
\hline Carbohydrate intake (g/day) & $225 \pm 72$ & $217 \pm 56$ & $225 \pm 56$ & $206 \pm 44 *$ \\
\hline Total dietary fibre intake (g/day) & $23 \pm 8$ & $23 \pm 7$ & $23 \pm 7$ & $22 \pm 7$ \\
\hline Insoluble & $15 \pm 5$ & $15 \pm 5$ & $15 \pm 5$ & $15 \pm 5$ \\
\hline Soluble & $7 \pm 2$ & $5 \pm 4 * * *$ & $7 \pm 5$ & $5 \pm 3 * * *$ \\
\hline Alcohol (g/day) & $9 \pm 15$ & $7 \pm 11$ & $10 \pm 16$ & $6 \pm 8 * * *$ \\
\hline
\end{tabular}

Data are mean \pm SD

Nutrient intake was calculated from 4 day food records

Significant change within the group: $* p \leq 0.05, * * * p \leq 0.001$. Individual variables did not differ significantly between groups 

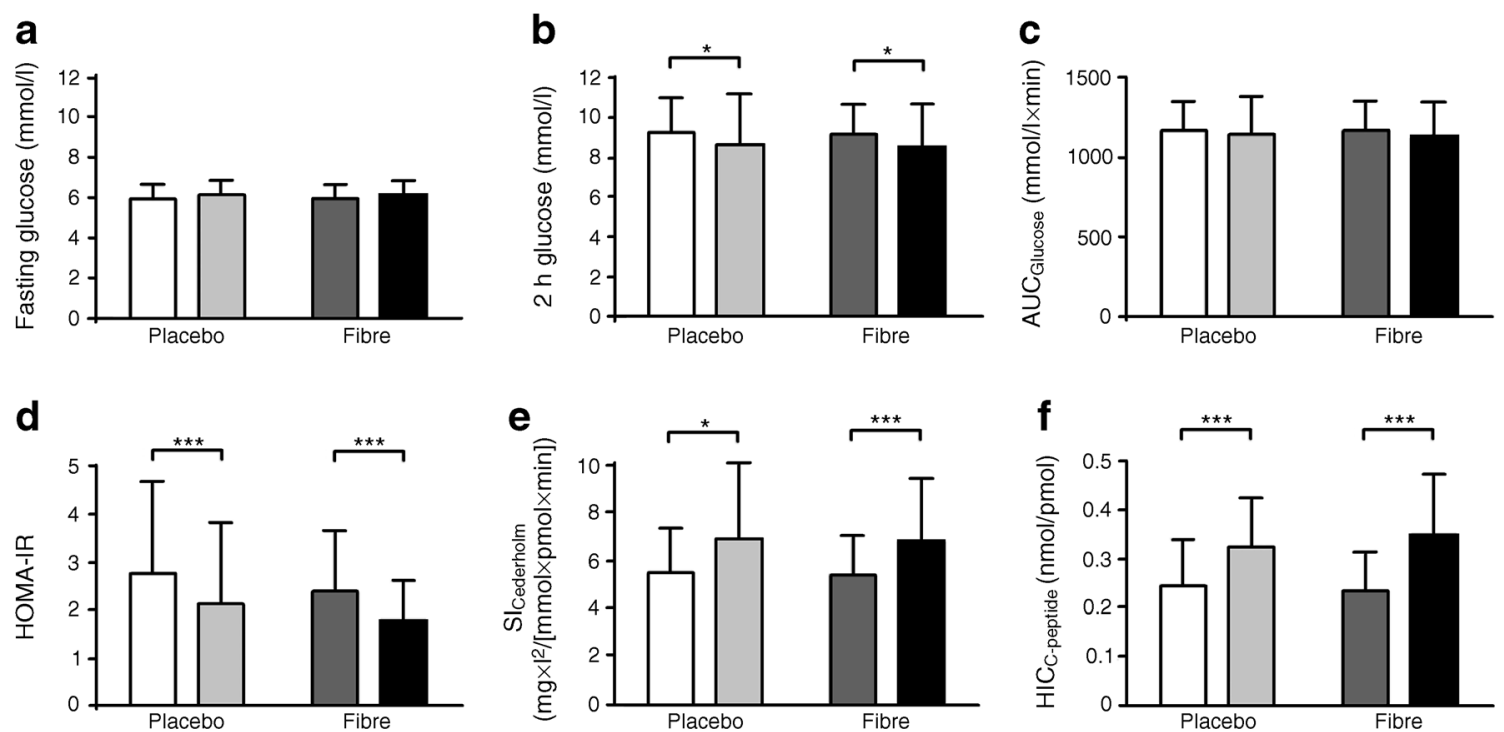

Fig. 4 Glycaemic control after 2 years of intervention (placebo group, $n=54$, fibre group, $n=52$ ). (a) Fasting glucose; (b) $2 \mathrm{~h}$ glucose; (c) $\mathrm{AUC}_{\mathrm{Glucose}}$; (d) fasting insulin sensitivity (HOMA-IR); (e) dynamic insulin sensitivity $\left(\mathrm{SI}_{\text {Cederholm }}\right)$; (f) $\mathrm{HIC}_{\mathrm{C} \text {-peptide }}$. White bars, placebo at

baseline; light grey bars, placebo after 2 years of intervention; dark grey bars, fibre at baseline; black bars, fibre after 2 years of intervention. Data are mean $\pm \mathrm{SD}$. Significant change within the group: $* p \leq 0.05$, $* * * \leq$ 0.001

$11.5 \pm 9.3 \mathrm{~g}$ to $34.4 \pm 8.3 \mathrm{~g}(p \leq 0.001)$. The total energy intake as well as the fat intake of the participants decreased during the dietary intervention in both groups. In the fibre group, also the mean protein and carbohydrate intake was significantly lower after the first 12 months (Table 2).

After the second year of the study, $2 \mathrm{~h}$ plasma glucose values were reduced in the fibre group by $-0.48 \pm$ $1.81 \mathrm{mmol} / \mathrm{l}(p=0.020)$ and in the placebo group by $-0.22 \pm 2.25 \mathrm{mmol} / \mathrm{l}(p=0.366)$ compared with baseline values (difference not significant). Surprisingly, the improvement in insulin sensitivity and HIC that could be observed after 1 year remained, only slightly diminished, in both groups after 2 years (Fig. 4), with no significant difference between the groups.

During the 24 month trial, diabetes was diagnosed in a total of 25 individuals - 16 in the placebo group and nine in the fibre group (Table 3). Thus, the incidence of diabetes in the fibre group was non-significantly $42-43 \%$ lower than in the placebo group.

\section{Discussion}

The main finding of our study is a significantly stronger reduction in $\mathrm{HbA}_{1 \mathrm{c}}$ and $2 \mathrm{~h}$ glucose levels (in women) after 1 year of supplementation with insoluble dietary fibre in individuals with IGT. Additionally, the incidence of diabetes was $42 \%$ lower in the fibre group, although this did not reach statistical significance. Although significant improvements in $\mathrm{HbA}_{1 \mathrm{c}}$ and glucose levels did not persist during the second year, the reduced incidence of diabetes in the fibre group was maintained.
Prevention of diabetes by lifestyle intervention has been investigated for several decades. Currently, the predominant approach is based on weight loss and increased physical activity. In these paradigms, dietary counselling includes low dietary total and saturated fat consumption, reduced cholesterol intake and increased fibre intake. With these approaches, recent lifestyle intervention programmes have been able to reduce the incidence of diabetes by $40-60 \%[1,2]$. Their counselling programmes were usually successful only with regard to weight loss and physical activity, whereas goals for fibre intake were achieved by as little as $25 \%$ of participants $[2,15,28]$. Thus, improving the intake of dietary fibre is one of the most taxing problems, as they are present in foods with

Table 3 Incidence of diabetes after the first and the second year

\begin{tabular}{llll}
\hline Variable & Baseline & 12 months & 24 months \\
\hline $\begin{array}{l}\text { Placebo } \\
\text { NGT/IFG/IGT }\end{array}$ & $91(100)$ & $61(87.1)$ & $48(75.0)$ \\
$\quad \begin{array}{l}\text { Type 2 diabetes } \\
\text { Fibre }\end{array}$ & - & $9(12.9)$ & $16(25.0)^{*}$ \\
NGT/IFG/IGT & $89(100)$ & $63(92.6)$ & $47(83.9)$ \\
Type 2 diabetes & - & $5(7.4)$ & $9(16.1)$ \\
& & $p=0.284^{\mathrm{a}}$ & $p=0.230^{\mathrm{a}}$ \\
\hline
\end{tabular}

Data are $n(\%)$ of participants

Classification was according to capillary blood sugar level

${ }^{\mathrm{a}} p$ values for the difference between the fibre and placebo group at the given time point (significant if $p \leq 0.05$ )

$* p \leq 0.05$, incident type 2 diabetes, placebo group, 24 months vs baseline IFG, impaired fasting glucose; NGT, normal glucose tolerance 
high caloric density, such as whole-grain bread, or are poorly accepted by participants due to taste (vegetables) or gastrointestinal side effects (grains and legumes).

Notably, the metabolic role of dietary fibre is controversial, as only insoluble fibre has been shown in epidemiological studies to be associated with a decreased risk of type 2 diabetes $[3,5,6,29]$. This is the first randomised intervention study demonstrating an improvement in $\mathrm{HbA}_{1 \mathrm{c}}$ over 1 year, a lower incidence of diabetes over 2 years and significantly reduced blood glucose levels upon intake of insoluble cereal fibre over 1 year in women. Similar studies have not been performed with either insoluble or soluble fibre. Therefore, the importance of solubility and fermentability of dietary fibre and the role of the microbiome in long-term improvements in blood glucose levels requires targeted studies [7].

We found a similar improvement in HIC and HOMA-IR in both groups. Weight loss tended to be slightly higher in the placebo group (without a statistically significant difference between the groups) and was almost non-existent at the end of the study, thereby excluding the hypothesis that the observed effects of insoluble cereal fibre were linked to weight changes [29]. Also, metabolic improvements did correlate with weight loss in the placebo group, but not in the fibre group, supporting independence of antidiabetic fibre effects from weight loss. These findings contradict recent studies showing greater weight loss with high-fibre diets, especially in individuals with impaired fasting glucose [30]. The metabolic effects were more pronounced and significant in the female subgroup, which may indicate sex-specific responses. Alternatively, the male group might have been too small.

As a strength of our study, the control group received the same lifestyle interventions known to improve glucose metabolism, but the significant effects of fibre supplementation could nevertheless be demonstrated. Evaluating dietary compliance, individuals in both groups were able to only slightly increase their fibre intake by conventional dietary improvements besides the supplement. Only $14.3 \%$ in the placebo group and $11.2 \%$ in the fibre group were able to achieve an average daily fibre load at the recommended level $(15 \mathrm{~g} / 4200 \mathrm{~kJ})$. When the supplement was included, $67.7 \%$ of the fibre group participants managed to achieve the recommendation. Therefore the significant additional improvement in the cereal fibre group supports the use of insoluble fibres as one component of diabetes prevention. In fact, insoluble fibres are widely available byproducts of flour production and could safely be added to numerous foods, thereby improving the population's cereal fibre intake. The absence of gastrointestinal side effects is another positive characteristic of this type of fibre, which underlines that fermentation is most likely not necessary for the beneficial effects on glucose metabolism $[8,29]$.

We experienced a dropout rate of $33 \%$, with an even distribution between the two study groups, which is an excellent outcome for a 2 year supplementation study and is comparable to other lifestyle intervention studies $[1,2,6,16,17]$.

Long-term observation after the end of dietary counselling demonstrated that dietary patterns again deteriorated rapidly and weight maintenance fell in most of the participants. Twohour glucose levels increased again in both study groups, although supplementation was continued during the 1 year follow-up period. We therefore conclude that a 1 year follow-up after the end of dietary counselling was sufficient to cover the main time frame of dietary rebound. Surprisingly, insulin sensitivity and HIC remained similar to the year before.

A placebo supplementation, especially in case of a study focusing on fibre intake, is very hard to develop. We decided to provide two supplements, which were equal in colour, digestibility, taste and caloric content to assure blinding over the entire study period and to avoid side effects by placebo or fibre supplement. It was considered the best option to use the slowly digestible carbohydrate isomaltulose as the main component of the placebo drink.

Limitations of the present study need mentioning. First, as is usual in most dietary trials, the sex distribution is skewed towards women, leading to a relatively small male subgroup, which then tends to be statistically unbalanced regarding body weight and glycaemic variables. However, by analysing the sexes separately, we were able to demonstrate that the pronounced effect in terms of glycaemic improvement was statistically significant in the large female subgroup, which was highly balanced regarding baseline anthropometric values.

Second, dietary compliance in terms of regular food intake and supplementation was controlled by self-reporting questionnaires. No biomarker is available to validate the reported fibre intake since alkylresorcinols (used to monitor cereal consumption) are not generated from insoluble cereal fibre. However, in general, we noticed an excellent correlation between reported energy intake and body weight changes, with an even distribution and only a few under- and over-reporters. We therefore assume that the dietary counselling was capable of stimulating acceptable dietary documentation from most volunteers.

Finally, there is no randomised control group, which would allow a precise estimation of the cumulative incidence of diabetes if there were no intervention at all. However, previous successful diabetes prevention trials have rendered the use of control groups without intervention unethical.

In summary, we cannot clearly support major benefits from a 2 year randomised placebo-controlled supplementation with insoluble low-digestibility dietary fibre on glycaemic metabolism, as all predefined outcomes failed to reach statistical significance. Effects on secondary outcomes, such as $\mathrm{HbA}_{1 \mathrm{c}}$ or $\mathrm{AUC}_{\mathrm{Glucose}}$, were pronounced in the first year, particularly in the more balanced female subgroup. Metabolic improvements in $2 \mathrm{~h}$ glucose levels were connected to improved 
fasting insulin sensitivity, insulin secretion and HIC. Fasting glucose levels did not change significantly.

Diabetes incidence was, non-significantly, $42-43 \%$ lower in the fibre group over the entire study period. This effect size is comparable with that of other lifestyle studies, which compared dietary counselling with sham intervention. Dietary counselling alone was not effective in increasing fibre intake in a significant and relevant manner, which highlights the need for fibre supplementation in order to achieve relevantly higher cereal fibre intake.

Supplementation did not lead to relevant gastrointestinal side effects, was accepted with moderate to high compliance and was associated with dropout rates comparable with those of other large lifestyle intervention studies with less intense dietary treatment $[1,2]$. Additionally, as a result of this, the participants were unable to identify their treatment allocation by self-observation of gastrointestinal side effects.

In modern developed societies, foods for which health claims are made are often more expensive and consumed predominantly by people with a higher awareness of healthy eating. Thus, people with low income or lower educational levels are not only at higher risk of becoming diabetic, but are also less likely to eat healthy food. It therefore seems both plausible and useful not to advertise fibre as an especially healthy component, but to supplement various types of food - cheap and expensive, for snacks and for regular eating - with dietary fibre.

Further studies are required to assess the need for and effectivity of supplementation with insoluble dietary fibre, as it is well accepted owing to its low rate and intensity of side effects and may still prove to be a promising way to decrease the incidence of diabetes on a population level.

Acknowledgements We thank our technical assistants and study nurses, in both the clinical wards and the laboratories, for their help in acquiring the study data and their crucial work with the participants.

Data availability Datasets are available by request from the corresponding author. Supplementary data is published as ESM. Some of the data were presented as an abstract at the 51st EASD Annual Meeting in 2015.

Funding General funding for this study was provided by the German Diabetes Foundation (grant no. 232/11/08; given to AFHP). Fibre and placebo supplements were provided by Rettenmaier \& Söhne, Holzmuehle, Germany. Neither funding party had any involvement in study design, data collection, data analysis, interpretation or writing of this publication.

Duality of interest SK and $\mathrm{CH}$ received a travel grant from Rettenmaier \& Söhne, Holzmuehle, Germany, including conference fees and accommodation. The authors declare no further conflicts of interest associated with this manuscript.

Contribution statement $\mathrm{CH}$ and SK wrote the paper, $\mathrm{CH}, \mathrm{CG}$ and $\mathrm{UD}$ conducted the experiments by dietary consultation, and collected and interpreted the resulting data. SK, MK and ALB performed the medical examinations and the medical supervision of the participants, and collected and interpreted the resulting data. $\mathrm{CH}, \mathrm{SK}$ and MAO performed the statistical analysis. CG, AMA, MOW and AFHP designed the study. All authors read and revised the manuscript, contributed to the discussion and approved the final version of this paper. SK is responsible for the integrity of the work as a whole and serves as guarantor of this work.

Open Access This article is distributed under the terms of the Creative Commons Attribution 4.0 International License (http:// creativecommons.org/licenses/by/4.0/), which permits unrestricted use, distribution, and reproduction in any medium, provided you give appropriate credit to the original author(s) and the source, provide a link to the Creative Commons license, and indicate if changes were made.

\section{References}

1. Knowler WC, Barrett-Connor E, Fowler SE et al (2002) Reduction in the incidence of type 2 diabetes with lifestyle intervention or metformin. N Engl J Med 346:393-403

2. Tuomilehto J, Lindstrom J, Eriksson JG et al (2001) Prevention of type 2 diabetes mellitus by changes in lifestyle among subjects with impaired glucose tolerance. N Eng1 J Med 344:1343-1350

3. Ley SH, Hamdy O, Mohan V, Hu FB (2014) Prevention and management of type 2 diabetes: dietary components and nutritional strategies. Lancet 383:1999-2007

4. Brombach C, Wagner U, Eisinger-Watzl M, Heyer A (2006) Nationale Verzehrsstudie II. Ernährungs-Umschau 53:4-9 document in German

5. Schulze MB, Schulz M, Heidemann C, Schienkiewitz A, Hoffmann K, Boeing H (2007) Fiber and magnesium intake and incidence of type 2 diabetes: a prospective study and meta-analysis. Arch Intern Med 167:956-965

6. de Munter JS, Hu FB, Spiegelman D, Franz M, van Dam RM (2007) Whole grain, bran, and germ intake and risk of type 2 diabetes: a prospective cohort study and systematic review. PLoS Med 4:e261

7. Isken F, Klaus S, Osterhoff M, Pfeiffer AF, Weickert MO (2010) Effects of long-term soluble vs. insoluble dietary fiber intake on high-fat diet-induced obesity in C57BL/6J mice. J Nutr Biochem 21:278-284

8. Weickert MO, Mohlig M, Koebnick C et al (2005) Impact of cereal fibre on glucose-regulating factors. Diabetologia 48:2343-2353

9. Robertson MD, Currie JM, Morgan LM, Jewell DP, Frayn KN (2003) Prior short-term consumption of resistant starch enhances postprandial insulin sensitivity in healthy subjects. Diabetologia 46: 659-665

10. Weickert MO, Mohlig M, Schofl C et al (2006) Cereal fiber improves whole-body insulin sensitivity in overweight and obese women. Diabetes Care 29:775-780

11. Weickert MO, Roden M, Isken F et al (2011) Effects of supplemented isoenergetic diets differing in cereal fiber and protein content on insulin sensitivity in overweight humans. Am J Clin Nutr 94:459471

12. Freeland KR, Wilson C, Wolever TM (2010) Adaptation of colonic fermentation and glucagon-like peptide-1 secretion with increased wheat fibre intake for 1 year in hyperinsulinaemic human subjects. Br J Nutr 103:82-90

13. Georg Jensen M, Kristensen M, Astrup A (2012) Effect of alginate supplementation on weight loss in obese subjects completing a 12wk energy-restricted diet: a randomized controlled trial. Am J Clin Nutr 96:5-13

14. Parnell JA, Reimer RA (2009) Weight loss during oligofructose supplementation is associated with decreased ghrelin and increased 
peptide YY in overweight and obese adults. Am J Clin Nutr 89: 1751-1759

15. Hermansen K, Hansen B, Jacobsen Ret al. Effects of soy supplementation on blood lipids and arterial function in hypercholesterolaemic subjects. Eur J Clin Nutr 2005; 59:843-850

16. Pan XR, Li GW, Hu YH et al (1997) Effects of diet and exercise in preventing NIDDM in people with impaired glucose tolerance. The Da Qing IGT and Diabetes Study. Diabetes Care 20:537-544

17. Ramachandran A, Snehalatha C, Mary S, Mukesh B, Bhaskar AD, Vijay V, Indian Diabetes Prevention Programme (IDPP) (2006) The Indian Diabetes Prevention Programme shows that lifestyle modification and metformin prevent type 2 diabetes in Asian Indian subjects with impaired glucose tolerance (IDPP-1). Diabetologia 49:289-297

18. Lindstrom J, Peltonen M, Eriksson JG et al (2006) High-fibre, lowfat diet predicts long-term weight loss and decreased type 2 diabetes risk: the Finnish Diabetes Prevention Study. Diabetologia 49:912920

19. Lindstrom J, Neumann A, Sheppard KE et al (2010) Take action to prevent diabetes - the IMAGE toolkit for the prevention of type 2 diabetes in Europe. Horm Metab Res 42(Suppl 1):S37-S55

20. Kulzer B, Hermanns N, Gorges D, Schwarz P, Haak T (2009) Prevention of diabetes self-management program (PREDIAS): effects on weight, metabolic risk factors, and behavioral outcomes. Diabetes Care 32:1143-1146

21. Hartmann BM, Vasquez-Caicedo AL, Bell S, Krems C, Brombach C (2008) The German nutrient database: basis for analysis of the nutritional status of the German population. J Food Compos Anal $21: 115-118$

22. Matthews DR, Hosker JP, Rudenski AS, Naylor BA, Treacher DF, Turner RC (1985) Homeostasis model assessment: insulin resistance and beta-cell function from fasting plasma glucose and insulin concentrations in man. Diabetologia 28:412-419

23. Katz A, Nambi SS, Mather K et al (2000) Quantitative insulin sensitivity check index: a simple, accurate method for assessing insulin sensitivity in humans. J Clin Endocrinol Metab 85:24022410

24. Belfiore F, Iannello S, Volpicelli G (1998) Insulin sensitivity indices calculated from basal and OGTT-induced insulin, glucose, and FFA levels. Mol Genet Metab 63:134-141

25. Aloulou I, Brun JF, Mercier J (2006) Evaluation of insulin sensitivity and glucose effectiveness during a standardized breakfast test: comparison with the minimal model analysis of an intravenous glucose tolerance test. Metabolism 55:676-690

26. Cederholm J, Wibell L (1990) Insulin release and peripheral sensitivity at the oral glucose tolerance test. Diabetes Res Clin Pract 10: 167-175

27. Pivovarova O, Bernigau W, Bobbert T et al (2013) Hepatic insulin clearance is closely related to metabolic syndrome components. Diabetes Care 36:3779-3785

28. Lindstrom J, Ilanne-Parikka P, Peltonen M et al (2006) Sustained reduction in the incidence of type 2 diabetes by lifestyle intervention: follow-up of the Finnish Diabetes Prevention Study. Lancet 368:1673-1679

29. Weickert MO, Pfeiffer AF (2008) Metabolic effects of dietary fiber consumption and prevention of diabetes. J Nutr 138:439-442

30. Hjorth MF, Ritz C, Blaak EE et al (2017) Pretreatment fasting plasma glucose and insulin modify dietary weight loss success: results from 3 randomized clinical trials. Am J Clin Nutr 106: 499-505 